\title{
Group Locomotor Imagery Training-Combined Knowledge of Performance in Community-Dwelling Individuals With Chronic Stroke: A Pilot Study
}

\author{
Bo-ram Choi $^{1}$, MSc, PT, Su-jin Hwang ${ }^{2}$, PhD, PT, Hee-won Lee ${ }^{1}$, MSc, PT, \\ Sun-young Kang ${ }^{1}$, MSc, PT, Hye-seon $\mathrm{Jeon}^{3}$, PhD, PT \\ ${ }^{1}$ Dept. of Physical Therapy, The Graduate School, Yonsei University, \\ ${ }^{2}$ Dept. of Physical Therapy, Nambu University, \\ ${ }^{3}$ Dept. of Physical Therapy, College of Health Science, Yonsei University, \\ Dept. of Ergonomic Therapy, The Graduate School of Health Science, Yonsei University
}

\begin{abstract}
We evaluated whether group locomotor imagery training-combined knowledge of performance (KP) lead to improvements in gait function in community dwelling individuals with chronic stroke. Ten adults who had suffered a hemiparetic stroke at least 6 months earlier participated in group locomotor imagery training-combined KP for 5 weeks, twice per week, with $2 \mathrm{~h}$ intensive training. Dynamic gait index scores increased significantly after the group locomotor imagery training-combined KP. However, times for the timed up-and-go test did not improve significantly after the training. Group locomotor imagery training-combined KP may be a useful option for the relearning of gait performance for community dwelling individuals with chronic hemiparetic stroke.
\end{abstract}

Key Words: Gait; Group training; Knowledge of performance; Locomotor imagery; Stroke.

\section{Introduction}

Stroke is the most common cause of adult disability, and $70 \sim 85 \%$ of first stroke survivors suffer from functional disabilities. Approximately 50 80\% of stroke survivors do regain some degree of gait performance, although most need to be involved in rehabilitation programs to achieve functional independence even 6 months after the stroke (Dobkin, 2003; Patel et al, 2000; Skilbeck et al, 1983). Thus, relearning gait function is one of the most important functional goals of post-stroke rehabilitation. Common gait-related impairments caused by stroke include decreased walking velocity, temporal asymmetry between more and less affected limbs, short stance phase, relatively longer swing phase in the more affected limb, and lack of overall gait performance. Decreased gait function is also related to the low level of physical fitness, and can ultimately result in difficulties in daily living activities (Hyndman and Ashburn, 2003; Perry et al, 1995).

The Bobath approach, strength training, intensive training, and body weight-supported treadmill training have been used to improve gait function in individuals following stroke (Dobkin, 2004; Ivey et al, 2008; Lindquist et al, 2007). Recently, small numbers of researchers have introduced the motor imagery training concept in stroke gait training (Bakker et al, 2008; Dickstein et al, 2004; Dunsky et al, 2006; Dunsky et al, 2008). Motor imagery is a cognitive operation that increases brain activity in neural cortical networks, and motor imagery practice is an active event in which the participant repeatedly imagines the performance of functional tasks or move-

Corresponding author: Hye-seon Jeon hyeseonj@yonsei.ac.kr 
ment routines without overt physical movements.

Motor imagery practice allows individuals with neurological disabilities to engage in meaningful and functional activities. Furthermore, motor imagery practice is a relatively low-cost and low-risk therapeutic intervention, and does not require much professional assistance, compared with other types of therapeutic intervention. Despite its advantages, some researchers have suggested shortcomings: specifically, that motor imagery practice does not identify whether a participant's performance is right or wrong, because neither the therapist nor the participant can observe any physical activity in motor imagery practice (Collet et al, 2011).

Knowledge of performance (KP) is an augmented feedback technique and provides information about movement characteristics that result in the performance outcome. KP provides performing information from which the patient can determine whether what he/she is doing is appropriate for performing the skill correctly, and is used for the person to compare his/her own performance against a performance goal. Thus, KP facilitates the achievement of the activities goal in the skill-learning process, and supplements the weaknesses of motor imagery practice (Schmidt and Lee, 2011).

The purpose of this study was to investigate the effects of group locomotor imagery training-combined $\mathrm{KP}$ on gait using the dynamic gait index (DGI), and the timed up-and-go (TUG) test to assess the associated performance level of motor and processing skills in gait performance of community dwelling in- dividuals who had suffered from chronic hemiparetic stroke. The hypothesis was that there would be differences between pretest and posttest in gait performance and related postural control variables in community dwelling individuals with chronic hemiparetic stroke.

\section{Methods}

This study was of a one-group pretest-posttest design with a 5-week intervention to examine the effects of $\mathrm{KP}-$ combined group locomotor imagery training, designed to improve participants' gait performance and to reduce gait disturbances, in community dwelling individuals with chronic hemiparetic stroke. They agreed to participate in the research and were physically able to access a group training room at a community health center by public transportation.

\section{Subjects}

In total, 10 community-dwelling adults with chronic hemiparetic stroke were recruited by convenience sampling from a community health center using a brochure containing study information in the city of Wonju. The study was approved by the human research ethics committee of Yonsei University in Wonju. All subjects signed an informed consent form after they received information about the study purpose, procedures, possible benefits and risks, privacy, and use of data.

Table 1. Characteristics of the participants

$(\mathrm{N}=10)$

\begin{tabular}{cc}
\hline \hline Variable & Participants \\
\hline Sex (male/female) & $8 / 2$ \\
Age (years) & $63.3 \pm 8.8^{\mathrm{a}}$ \\
Post-stroke duration (months) & $60.0 \pm 40.5$ \\
Etiology (ischemic/hemorrhage) & $7 / 3$ \\
Paretic side (right/left) & $6 / 4$ \\
Brunnstrom stage (stage 3/stage 4) & $1 / 9$ \\
\hline
\end{tabular}

\footnotetext{
${ }^{a}$ mean \pm standard deviation
} 
Table 2. Items in dynamic gait index

\begin{tabular}{c}
\hline \hline Items \\
\hline Steps \\
Vertical head turns \\
Stepping over obstacle \\
Gait on level surface \\
Gait with pivot and stop \\
Gait with speed changes \\
Stepping around obstacle
\end{tabular}

Inclusion criteria were (1) at least 6 months since stroke onset, (2) ability to walk independently over ground for at least $10 \mathrm{~m}$ with or without use of an ankle-foot orthosis or assistive device without a break, (3) absence of any cognitive impairment (scores above 24 on the Mini Mental State Examination), (4) ability to understand verbal instructions, (5) no cerebellar lesion, (6) no significant body or visuospatial hemineglect, (7) first stroke survivors, and (8) good imagery functioning (a score of 32 or higher on the revised Movement Imagery Questionnaire) (Hwang et al, 2010). Table 1 shows the general characteristics of the participants.

\section{Instruments}

Functional gait performance and related postural control variables were assessed using clinical measures, the DGI, and the TUG test. The DGI was de- veloped to evaluate and document a patient's ability to modify gait in response to changing task demands during ambulation. The test involves eight items on four-point scales (0 [severe impairment] to 3 [normal]) (Shumway-Cook et al, 1997). The reliability and validity of the DGI in stroke populations has been reported to be excellent (Jonsdottir and Cattaneo, 2007). The TUG test measures the timing component of gait performance while a subject stands up from a chair, walks $3 \mathrm{~m}$, turns around, and returns to the chair. It examines primarily proactive or anticipatory aspects of postural control associated with rising from a chair, turning, and sitting down. Neurologically impaired adults who took longer than $30 \mathrm{~s}$ to complete the test were dependent in most activities of daily living and mobility skills. The test has been reported to be reliable and valid for examination of mobility following stroke (Faria et al, 2009; Ng and Hui-Chan, 2005).

\section{Interventions}

The subjects participated in 5 weeks of group lo- $^{-}$ comotor training-combined KP twice per week, with each session lasting $2 \mathrm{~h}$, by performing a five-stage protocol: progressive relaxation, external imagery (analysis of task sequences), problem identification, internal imagery, and mental rehearsal. Table 3 shows the definition, purposes, and methods of the

Table 3. The five-stage protocol of $\mathrm{KP}^{-}$-combined group locomotor imagery training

\begin{tabular}{|c|c|c|}
\hline Stage & Purpose & Method \\
\hline $\begin{array}{c}\text { Stage I } \\
\text { Progressive relaxation stage }\end{array}$ & to increase blood flow to the brain & Benson's relaxation protocol \\
\hline $\begin{array}{c}\text { Stage II } \\
\text { External imagery stage }\end{array}$ & $\begin{array}{l}\text { to analyze the gait cycle's sequences } \\
\text { to understand the subject's own gait }\end{array}$ & Normal modeling using videotape \\
\hline $\begin{array}{c}\text { Stage III } \\
\text { Problem identification stage }\end{array}$ & $\begin{array}{c}\text { dysfunction and to compare their } \\
\text { gait with that of a normal young } \\
\text { adult }\end{array}$ & $\begin{array}{l}\text { Knowledge of performance using } \\
\text { videotape of patients' own gait }\end{array}$ \\
\hline $\begin{array}{c}\text { Stage IV } \\
\text { Internal imagery stage }\end{array}$ & $\begin{array}{l}\text { to plan and image the gait } \\
\text { performance in first-person } \\
\text { perspective }\end{array}$ & Normal modeling using videotape \\
\hline $\begin{array}{c}\text { Stage } \mathrm{V} \\
\text { Mental rehearsal stage }\end{array}$ & $\begin{array}{l}\text { to image the normal gait } \\
\text { performance }\end{array}$ & Mental replay \\
\hline
\end{tabular}


five-stage protocol.

In this experiment, two different videotapes were used. The first was a recording of normal gait to provide the idea of normal gait to maximize internal and external imagery in the group locomotor imagery training. We recorded normal ambulation from anterior, posterior, and side views while a 20-year-old healthy male walked slowly for $6 \mathrm{~min}, 23 \mathrm{~s}$ and then at normal speed for $4 \mathrm{~min}, 44 \mathrm{~s}$. For the problem-identification stage, we used the second videotape to provide KP. The contents of this videotape was each patient's own gait performance from anterior, posterior, and side views while walking along the midline of a $10 \mathrm{~m}$ walkway.

\section{Procedures}

All test and training procedures in this community-based group program were conducted in the group-training room of a community health center. Before starting the 5-week motor imagery training (upon initial evaluation), we videotaped each subject's gait performance for the KP. This videotape was used for the problem identification stage in the first 2 weeks of training. On the first day of the third training week, we videotaped the patients' own gait performance again to update the content for the KP for the remaining training sessions. The level of gait performance in each participant was evaluated twice by means of the DGI, and TUG test, on the days before and after the training. Participants underwent the group locomotor imagery training-combined KP according to the five-stage protocol for $2 \mathrm{~h}$ per session under the supervision of three well-trained physical therapists (Table 2). The first week of the 5-week training focused on familiarizing patients with normal gait sequences and identifying their own problems in gait by studying the differences between the performance of the subject's gait and the normal gait using video replay. A 5 min break was provided between stages III and IV.

\section{Statistical Analysis}

Wilcoxon matched-pairs, signed-ranks tests were used to compare the scores of the DGI, and TUG test prior to and after the group locomotor imagery training. The significance level was set at $a=.05$. All statistical analyses were performed using the SPSS ver. 18.0 software.

\section{Results}

Figure 1 shows a comparison of pretest and posttest DGI scores. DGI scores increased significantly after the training $(\mathrm{p}<.05)$. The times for the timed up-and-go test did decrease after training, compared with before training, but not statistically significantly so $(\mathrm{p}<.05)$ (Figure 2).

\section{Dynamic Gait Index}

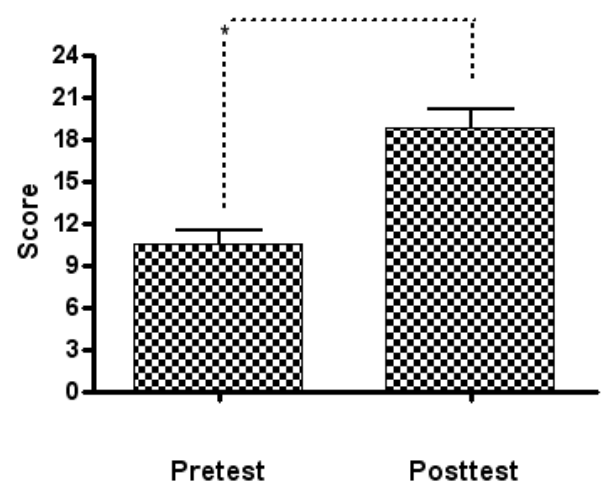

Figure 1. The score of dynamic gait index $\left({ }^{*} \mathrm{p}<.05\right)$.

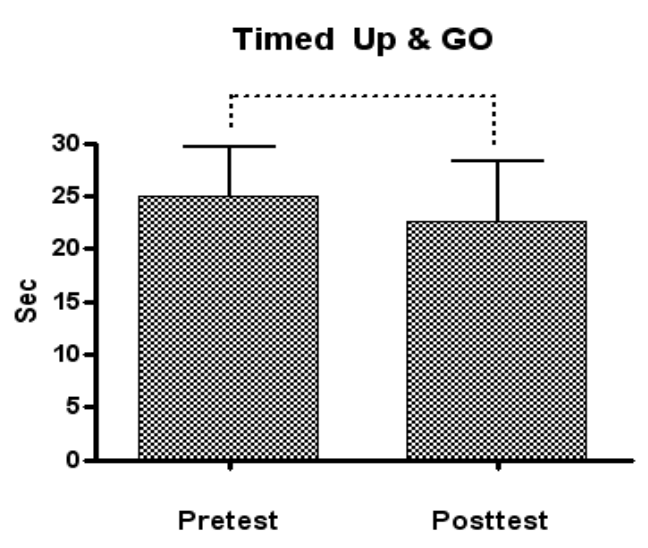

Figure 2. The score of timed up-and-go. 


\section{Discussion}

The purpose of this study was to investigate the effects of group locomotor imagery training-combined $\mathrm{KP}$ on balance and gait performance in community-dwelling individuals with chronic hemiparetic stroke. Two clinical tools were used to determine whether the intervention affected gait-related function. Our results revealed that the DGI scores increased significantly after the KP-combined group locomotor imagery training. However, the times for the TUG test were not improved significantly after the training.

Improvements in DGI scores after the intervention likely mean that the intervention was generally beneficial in improving the gait abilities of chronic hemispheric stroke patients. The DGI score is known as a predictor of fall risk in patients with neurological disorders as well as healthy older adults (Herman et al, 2009; McConvey and Bennett, 2005). All items in the DGI were designed to test ambulation function when the subject is standing upright. Although the test items include some secondary task situations, such as changing velocity and dealing with stairs, in addition to pure ambulation, the DGI does not include any other functional component, such as sit-to-stand or stand-to-sit (Table 2). In contrast, the TUG test starts with sit-to-stand activity from a chair and ends with a stand-to-sit action. Also, the TUG test includes turning all the way around while walking.

Our training protocol was very specific to basic ambulation itself, and it did not include any other functional task. The lack of a statistically significant improvement in TUG with a significant improvement in DGI after the group locomotor imagery training-combined KP may be explained, at least partially, by the specificity of the training. Thus, the likelihood of functional changes after the group locomotor imagery training-combined $\mathrm{KP}$ was higher in the DGI score than in the TUG test. As noted by much motor-learning-related literature, the transfer effect of a learning gait task is relatively small in terms of TUG results.

Most importantly, the results of this study partially support the feasibility of group-centered locomotor imagery training for the community-dwelling chronic hemiplegic population; it was found to be as effective as individual training. The first advantage of group locomotor imagery training-combined KP is the enjoyment of a group exercise instead of the boredom that often comes with individual training. Group training may facilitate motivation and encourage individual participants to achieve goals during enjoyable group interactions (Pang et al, 2006). Additionally, cost-effectiveness is another advantage of group-centered training. Recently, cost reduction has become a major issue in healthcare and consequently the paradigm has shifted from the clinic to the home and community settings and from an individual to a small group basis, which has reduced supervision time (Eng et al, 2003).

In this study, we showed that group locomotor imagery training could be a safe treatment option with low fall and fall-related injuries for the chronic hemiplegic population with gait disturbances. However, there are several limitations to this study. First, we did not include a control group. Thus, we could not assess whether the addition of videotaped $\mathrm{KP}$ on top of classical imagery training was more effective in improving gait. Second, because this was an off-campus community-based training program with limited time and human resources for pre $^{-}$and post-training evaluation, we did not measure any biomechanical or spatiotemporal parameter. Third, our training protocol may have been too specific for ba ${ }^{-}$ sic gait patterns alone. To improve the overall functional independence of individuals with chronic hemiplegia in the community, group-imagery training protocols should include other functional activities as well as basic gait patterns. Thus, on the basis of the findings of this preliminary study, a case control study with a larger sample size that incorporates various functional tasks in the training protocol 
should be conducted

\section{Conclusion}

In this one-group pretest-posttest design study with a 5-week intervention, we examined the effects of group locomotor imagery training-combined $\mathrm{KP}$ designed to improve gait performance and to reduce gait disturbances in community-dwelling individuals with chronic hemiparetic stroke. The results of this preliminary study suggested that the 5 weeks of group locomotor training-combined KP improved gait performance in the community-dwelling chronic hemiplegic population. The novelty of this pilot study lies in the imagery training being provided as a group session, and a videotaped KP of their own gait performance was given to each participant. However, future well-designed studies are necessary to confirm our conclusions.

\section{References}

Bakker M, Overeem S, Snijders AH, et al. Motor imagery of foot dorsiflexion and gait: Effects on corticospinal excitability. Clin Neurophysiol. 2008;119(11):2519-2527.

Collet C, Guillot A, Lebon F, et al. Measuring motor imagery using psychometric, behavioral, and psychophysiological tools. Exerc Sport Sci Rev. 2011;39(2):85-92.

Dickstein R, Dunsky A, Marcovitz E. Motor imagery for gait rehabilitation in post-stroke hemiparesis. Phys Ther. 2004;84(12):1167-1177.

Dobkin BH. Strategies for stroke rehabilitation. Lancet Neurol, 2004;3(9)528-536.

Dobkin BH. The Clinical Science of Neurologic Rehabilitation. 2nd ed. New York, Oxford University Press, 2003:58-63.

Dunsky A, Dickstein R, Marcovitz E, et al. Motor imagery practice in gait rehabilitation of chronic post-stroke hemiparesis: Four case studies. Arch Phys Med Rehabil. 2008;89(8):1580-1588.

Dunsky A, Dickstein R, Ariav C, et al. Home-based motor imagery training for gait rehabilitation of people with chronic poststroke hemiparesis. Int J Rehabil Res. 2006;29(4):351-356.

Eng JJ, Chu KS, Kim CM, et al. A community-based group exercise program for persons with chronic stroke. Med Sci Sports Exerc. 2003;35(8):1271-1278.

Faria CDC, Teixeira-Salmela LF, Nadeau S. Effects of the direction of turning on the timed up \& go test with stroke subjects. Top Stroke Rehabil. 2009;16(3):196-206.

Herman T, Inbar-Borovsky N, Brozgol M, et al. The dynamic gait index in healthy older adults: The role of stair climbing, fear of falling and gender. Gait Posture. 2009;29(2):237-241.

Hwang S, Jeon HS, Yi CH, et al. Locomotor imagery training improves gait performance in people with chronic hemiparetic stroke: A controlled clinical trial. Clin Rehabil. 2010;24(6):514-522.

Hyndman D, Ashburn A. People with stroke living in the community: Attention deficits, balance, ADL ability and falls. Disabil Rehabil. 2003;25(15):817 -822 .

Ivey FM, Hafer-Macko CE, Macko RF. Task-oriented treadmill exercise training in chronic hemiparetic stroke. J Rehabil Res Dev. 2008;45(2): 249-259.

Jonsdottir J, Cattaneo D. Reliability and validity of the dynamic gait index in persons with chronic stroke. Arch Phys Med Rehabil. 2007;88(11):1410 -1415 .

Lindquist AR, Prado CL, Barros RM, et al. Gait training combining partial body-weight support, a treadmill, and functional electrical stimulation: Effects on poststroke gait. Phys Ther. 2007;87(9):1144-1154.

Ng SS, Hui-Chan CW. The timed up \& go test: Its reliability and association with lower-limb impairments and locomotor capacities in people 
with chronic stroke. Arch Phys Med Rehabil. 2005;86(8):1641-1647.

McConvey J, Bennett SE. Reliability of the dynamic gait index in individuals with multiple sclerosis. Arch Phys Med Rehabil. 2005;86(1):130-133.

Pang MY, Harris JE, Eng JJ. A community-based upper-extremity group exercise program improves motor function and performance of functional activities in chronic stroke: A randomized controlled trial. Arch Phys Med Rehabil. 2006;87(1):1-9.

Patel AT, Duncan PW, Lai SM, et al. The relation between impairments and functional outcomes poststroke. Arch Phys Med Rehabil. 2000;81(10): 1357-1363.

Perry J, Garrett M, Gronley JK, et al. Classification of walking handicap in the stroke population. Stroke. 1995;26(6):982-989.

Schmidt RA, Lee TD. Motor Control and Learning: A behavioral emphasis. 5th ed. Champaign, IL, Human Kinetics, 2011:372-377.

Shumway-Cook A, Baldwin M, Polissar NL, et al. Predicting the probability for falls in community-dwelling older adults. Phys Ther. 1997;77(8):812-819.

Skilbeck CE, Wade DT, Hewer RL, et al. Recovery after stroke. J Neurol Neurosurg Psychiatry. 1983;46(1):5-8.

This article was received January 6, 2013, was reviewed January 8, 2013, and was accepted February 1, 2013 